\title{
Human Identification Based on Hand Dorsal Vein Pattern using BRISK \& SURF Algorithm
}

\author{
Aryan Singh, Hardik Goyal, Amar, Ajai Kumar Gautam
}

\begin{abstract}
Security is an essential part of our lives today as people have many accounts and each account generally has a high amount of transactions. Biometrics are in the trend as it reduces risk and is quite difficult to replicate. Currently most popular biometrics such as fingerprint, retina can be forged. Dorsal Vein pattern is gaining attention these days due to its contactless nature and difficult to forge. These vein patterns can give rise to another reliable biometric which is better than the biometrics being currently used. In this paper, we have discussed the technique to extract the important information in the form of points and use them for analysis based on different parameters. The analysis will help us to know the reliability of biometrics using hand dorsal vein pattern. We have used SUAS Dorsal Hand Vein Database which is available online [18]. We have implemented the technique on MATLAB - R2019 image processing toolbox. We have used end points and branch points of the vein pattern to form triplets using Delaunay's Triangulation. Then these triplets are matched using proposed matching technique. We have used matching techniques in MATLAB for analysis. We have used the BRISK and SURF algorithm for matching. The objective of this paper is to build a distinct and a substitute to the contact-based systems. Vein pattern biometrics are quite useful in reducing crimes as it is a contactless biometric.
\end{abstract}

Index Terms: Biometrics, Hand Dorsal Veins, Region of interest, End Points and Branch Points, Matching, FAR.

\section{INTRODUCTION}

Hand vein pattern is a huge network of blood streams under a person's skin. Hand vein patterns are classified into hand dorsal vein pattern and hand palm vein pattern. Hand dorsal vein patterns are unique for every individual, even twins do not have the same hand dorsal vein pattern. So, this quality of uniqueness is essential to construct a biometric system using the hand dorsal vein pattern.In this digital era biometrics are one of the advanced tools to combat the cyber security risk. Today, most of the biometrics are contact based such as fingerprint, retina etc. These biometrics parameters are easily traceable and did not offer high reliability. Also, hand veins inside the skin temperature does not have any effect on the efficiency of the biometric system.

Revised Manuscript Received on April 25, 2020.

* Correspondence Author

Aryan Singh*, Department of Electronics and Communication Engineering, Delhi Technological University, New Delhi, India.

Hardik Goyal, Department of Electronics and Communication Engineering, Delhi Technological University, New Delhi, India.

Amar, Department of Electronics and Communication Engineering, Delhi Technological University, New Delhi, India.

Ajai Kumar Gautum, Department of Electronics and Communication Engineering, Delhi Technological University, New Delhi, India.

(C) The Authors. Published by Blue Eyes Intelligence Engineering and Sciences Publication (BEIESP). This is an open access article under the CC BY-NC-ND license (http://creativecommons.org/licenses/by-nc-nd/4.0/)
While hand dorsal vein pattern biometrics is a contactless biometric system. There is a high demand for an affordable, reliable and unique biometric system to identify each individual without much error. Also, Biometric identification is giving competition to other authentication systems such as PIN, password, RFID [1].

\section{LITERATURE REVIEW}

In the past few years, there has been a regular pattern to construct a human identification using the dorsal vein pattern. In this Biometric process. First step is image capturing, then image processing, region of extraction, feature extraction then matching and classification. Image capturing is done through an infrared camera because infrared images provide better contrast. Badawi have used infrared images using CCD (Charged Couple Device) video camera [4].We have used SUAS Dorsal Hand Vein Database [18]. In Image Processing, normalization process is common nowadays. Sathish used normalization technique for extracting the hand vein pattern. Adaptive histogram equalization technique is also popular now [15]. Contrast limited adaptive histogram equalization (CLAHE) has been used by Abdul Kareem Z. Mohammed [16]. Then, noise will be removed by using various filters such as Gaussian filters, median filters.Region of Interest (ROI) Extraction is a very important part of the biometric process. Region of interest includes the hand vein pattern only. Abdul Kareem Z. Mohammed used the whole dorsal hand [16]. While Marlina Yakno uses point-based hand vein pattern extraction [6]. This point-based extraction technique is a new technique and helpful in getting good matching percentage as shown later in this paper.In feature extraction, Sumit Kulkarni uses thinning of the region of extraction to 1pixel size, then extract the branch and end points from the thinned image [1]. Pooja Ramsoful has proposed three feature extraction techniques [11]. First is the Hough transform method which detects straight lines in a thinned image She has used probabilistic Hough Line Transform as it is an enhanced version of Hough Line Transform [11]. This transform allows easy comparison between images. Second one is Pixel by Pixel Method, which is the same used by Sumit Kulkarni [1]. While the third one is Directional coding method in which First, Wavelet Transform is applied on the image to break up the hand vein image into a reduced resolution image [11]. A lot of matching and classification techniques have been used earlier.

Published By:

Blue Eyes Intelligence Engineering \& Sciences Publication 
Classifiers such as Euclidean, SVM, KNN, Random Forest Classifiers are quite common. M. Rajalakhmi have used CNN for matching and analysis [17]. C. Premavathi have used KNN classification technique [19]. While Ricardo Janeshas used random forest classifier and uses his results to compare random forest classifier with other matching techniques[10].Concept of FAR (False Acceptance Rate) which is the percentage of identification instances in which unauthorized persons are incorrectly accepted and FRR (False Rejection Ratio) the percentage of identification instances in which authorized persons are incorrectly rejected have been used by Tarshi Jain [3]. This concept is also used by Ricardo Janes to compare different matching techniques [10].

\section{PROPOSED METHODOLOGY}

The proposed framework for Hand Dorsal vein pattern-based authentication system comprises of six modules each complying to a different purpose:
A. IMAGE DATA ACQUISITION
B. KNUCKLE MASK CREATION
C. REGION OF INTEREST EXTRACTION
D. PRE-PROCESSING
E. FEATURE EXTRACTION
F. MATCHING ALGORITHM

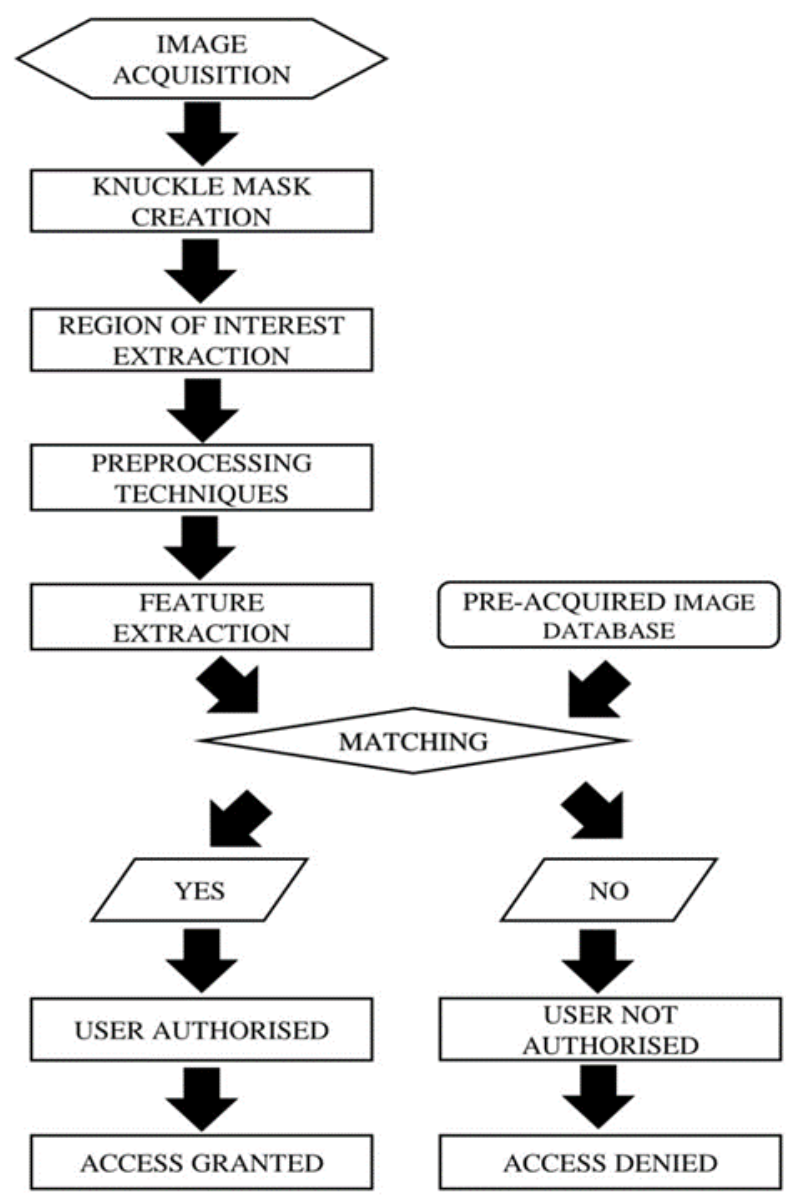

Fig.1 Block Diagram of the System

\section{A. IMAGE DATA ACQUISITION}

We have used SUAS (Sakarya University of Applied Sciences) Dorsal Hand Vein Database containing 919 images in which 155 adults (80 males, 75 females) participated in the study [18]. Subjects were asked to place their right and left hands at first position under the infrared camera on a white surface for 3 seconds. The infrared light waves penetrate the skin and darken the veins due to the absorption of light by haemoglobin pigment present in blood [13]. The 700 to 900 nanometres wavelength light rays are sensible to haemoglobin [1][3][5][10]. Three types of vein patterns exist in hand i.e. Palm veins, Finger veins and Dorsal veins. We have focused on Hand Dorsal veins. The dataset is divided into subsets of 3 images per hand or 6 images per person captured at different orientations of placement on white surface. The resolution of each image is $640 \times 480$. We have used Hand Dorsal Veins in this paper.

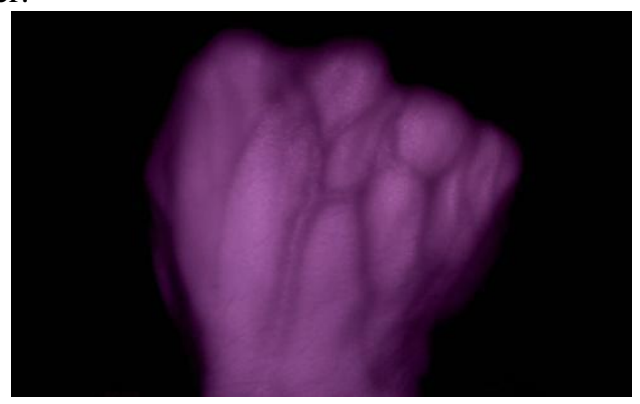

Fig.2 Image acquisition

\section{B. KNUCKLE MASK CREATION}

In the first step of the process, the image is converted from RGB to grayscale. Then, the grayscale image is Binarized at a Threshold level computed using Otsu's method [1][13][16]. Otsu's method gives a level for thresholding pixels by choosing the minimum variance between black and white pixels at which they become identifiable [2]. The pixels closer to black are assigned 0 value and the ones nearer to white are given value 1.Now, the generated mask outline contains some scattered particles and imperfections which are rectified using image dilation, small element filtering and image erosion. An optimum morphological structuring element is chosen for these operations which alters the outline edge pixels according to neighbouring pixel pattern [12]. We used line and disk structuring elements for best results. This generated mask becomes the basis of Region of Interest Extraction.

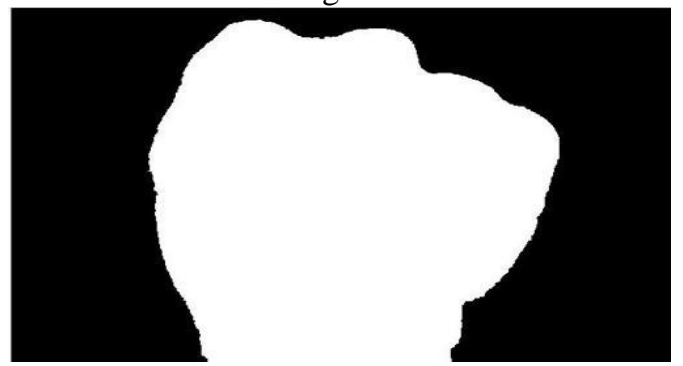

Fig.3 Knuckle Mask Creation

Published By:

Blue Eyes Intelligence Engineering \& Sciences Publication

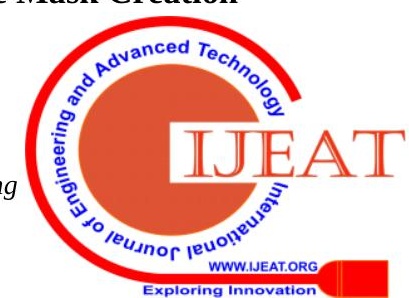




\section{REGION OF INTEREST (ROI) EXTRACTION}

The acquired hand knuckle images are now cropped adaptively and precisely in order to extract the area where the highest density of nerve pattern is present. The generated mask is superimposed on the acquired image in-order to define the knuckle boundary required for cropping the ROI.A challenge arises in this process due to loss of veins present at the edges of the hand knuckle. Many researchers have proposed different methods of ROI selection previously [16]. We have used 2 progressive methods for ROI extraction:

1. Bounding Box Cropping

2. Point Mapped Cropping

1. BOUNDING BOX CROPPING

Firstly, we have used regionprops built in function of MATLAB vR2019a which returns a set of properties specified for each 8- connected component (object) in the binary image $[21,22]$. We have used the "BoundingBox" property which returns the dimensions of the smallest rectangle that can enclose the hand knuckle in our case. The reduction of Non-useful background helps in better mapping of points in the next process of ROI creation.

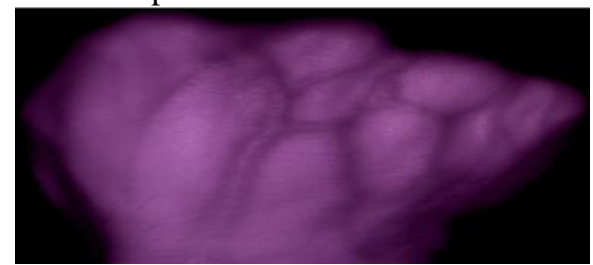

Fig.4 Bounding Box Cropping

2. POINT MAPPING METHOD

This method firstly involves tracing the boundary of the generated binary mask [4][6]. A reference point is chosen such that it lies in the middle of the bottom edge of mask and Euclidean Distance is computed between the reference point and the boundary points one by one [6].Each boundary point's distance is measured and a distance distribution graph is plotted between the position of boundary point (Boundary Point Vectors) versus distance computed [4][6].The Local Maximas indicate the finger joints and the Local Minimas indicate the gaps between the joints. These points are used to adaptively crop a Region of interest (ROI). This method also works with different orientations of hand placement on the surface.

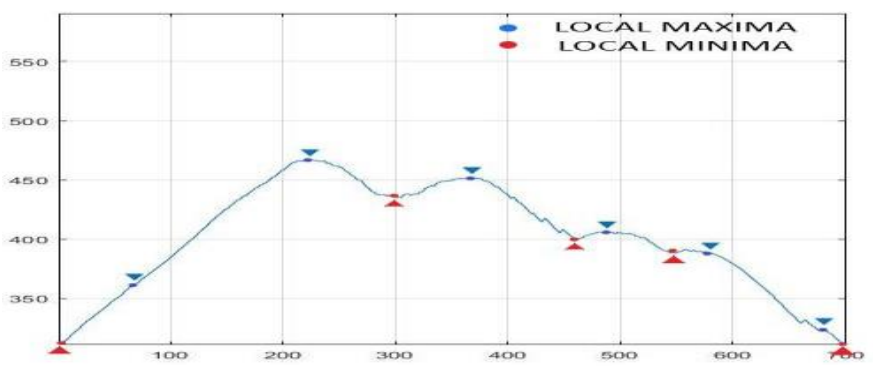

Fig.5 Feature points detection

The above graph represents a shape of a dorsal hand in of minima and maxima. Y-Axis on the graph represents Distance from the reference point of the wrist while X-Axis represents the position of the pixel in the contour. These points along with bounding box cropped image is used to detect the effective ROI of the hand dorsal vein pattern.

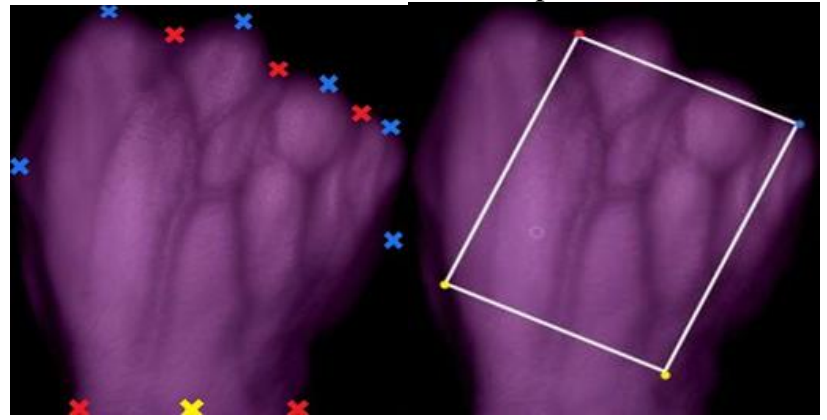

Fig.6a Points mapped according to graph obtained Fig.6b Detected ROI in Image

The extracted points are superimposed on the cropped Bounding Box figure which are shown by three different colour schemes. Blue points refer to finger joints (Local Maximas), Red ones refer to space between joints (Local Minimas) \& yellow one is the Bottom Reference Median Point. The maximum area enclosing rectangle is formed using different combinations of four corner points.

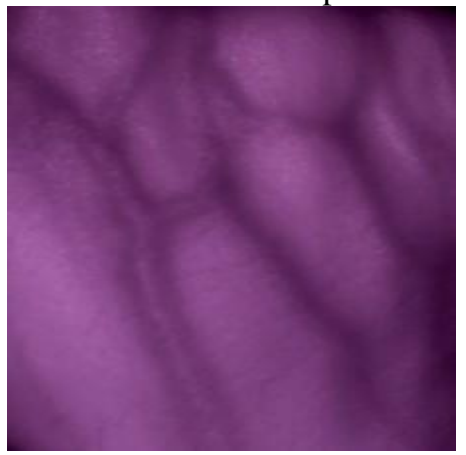

Fig.7 Cropped Image

\section{PRE-PROCESSING TECHNIQUES}

After cropping the Region of Interest (ROI) from the test image we have applied image filtering MATLAB functions to enhance the vein pattern distinctively [20].Blurriness and noise are added to the image due to illumination while it is being captured by the Near Infrared Camera [7][8][9]. We have used two types of filters to combat this issue.

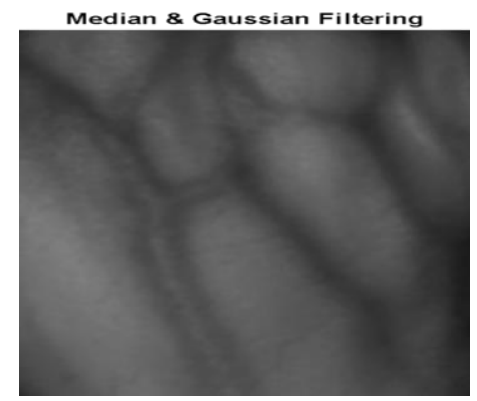

Fig.8 Filtering

Firstly, Median Filter is used then Gaussian Filtration is performed. Median Filter removes salt \& pepper noise from the image by assigning every pixel the median value of the

Published By:

Blue Eyes Intelligence Engineering \& Sciences Publication

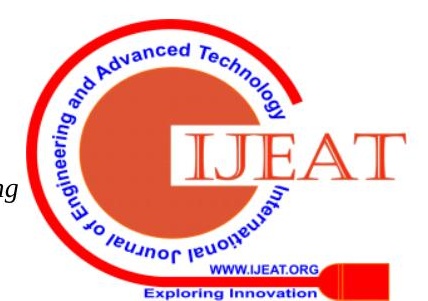


surrounding 9 pixels neighbourhood. Gaussian Filter helps in further smoothening of image for better image processing without any information loss [11]. Filtering \& Contrast Enhancement together constitute towards Pre-processing in this Biometric system [8].Now, the contrast of the image has to be enhanced as the captured image's contrast is quite less [1][7]. Higher contrast helps in better identification of veins in the captured image. This function can be performed in three ways: -

i. Histogram Equalization (HISTEQ)

ii. Contrast Limited Adaptive Histogram Equalization (CLAHE)

\section{i. HISTOGRAM EQUALIZATION}

Histogram equalization revolves around transforming the intensity values of the input image so that the histogram of the output image approximately matches a pre assigned histogram [22]. This method produces an output image with pixel values evenly distributed throughout the range by matching a flat histogram with 64 bins histogram [21][22].

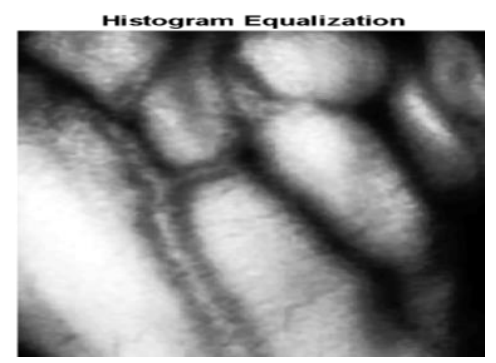

Fig.9 Histogram Equalization

The output image shows improvement in contrast but veins present in the corner and left side are still not significantly distinguishable from the background skin.

\section{ii. CONTRAST LIMITED ADAPTIVE HISTOGRAM EQUALIZATION(CLAHE)}

The CLAHE algorithm, adapthisteq operates on small regions in the image, called tiles instead of working on the entire image like histeq [22]. CLAHE enhances the contrast of each individual tile, so that the histogram of the output region approximately matches a pre assigned histogram [22]. We have used $8 \times$ x 8 tile size specified by 'NumTiles' parameter.Moreover, after performing the equalization, CLAHE combines the tiles in each respective neighbourhood using bilinear interpolation to eliminate any induced artificial boundaries [22].

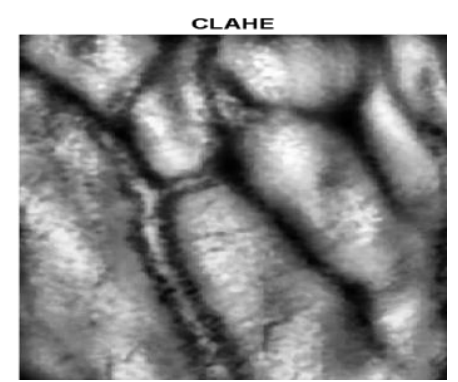

Fig.10 Adaptive Histogram Equalization

Thus, the contrast is now adequately enhanced making each vein distinctive from the skin. We have used 'ClipLimit' with value 0.05 and 'Distribution As exponential parameter in

\section{CLAHE function to achieve maximum refinement.}

\section{E. FEATURE EXTRACTION}

After enhancing contrast \& nullifying noise from the image, we focus on obtaining the vein pattern features. This process involves image thresholding, Dilution, small element(blob) removal and 1-bit Morphological Thinning called skeletonization [8][9][11].

At last the features extracted from the vein pattern are endpoints and bifurcation points [9]. Endpoints are the points where veins end and the bifurcation points are points where veins are divided into different branches [9].

\section{i. ADAPTIVE THRESHOLDING}

The adaptthreshMATLAB function computes a locally adaptive threshold for a grayscale image [9][22]. It chooses the threshold based on the local mean intensity in the $3 \times 3$ neighbourhood of each pixel. The computed threshold along with sensitivity factor is used to binarize the image [22]. Sensitivity factor is chosen in the range $(0,1)$ that indicates sensitivity towards thresholding more pixels as foreground [22]. We concluded 0.79 as the best performing value of sensitivity factor in our case.

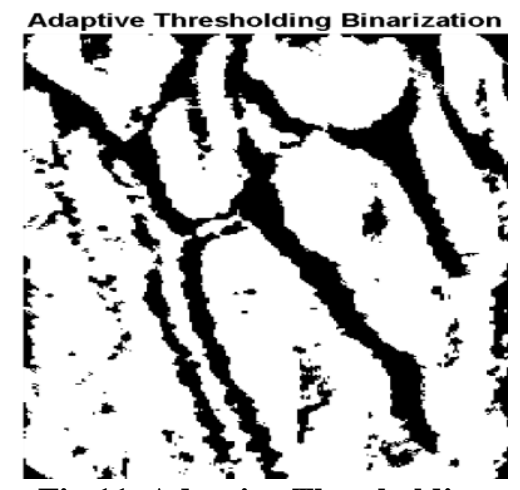

Fig 11. Adaptive Thresholding

\section{ii. ROI DILUTION}

The image is complemented and a rectangular morphological structuring element is so chosen to obtain an output image that contains each distinctive vein within a connected pattern. Some blobs are still present which are completely removed in the next process. Dilution involves averaging of pixel values in objects in accordance to the corresponding neighbourhood.

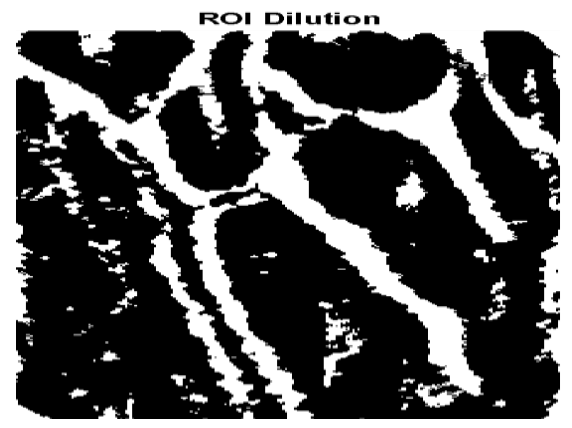

Fig.12 Dilation

Published By:

Blue Eyes Intelligence Engineering \& Sciences Publication 
iii. SMALL ELEMENT FILTERING

We have used bwareafilt MATLAB function to remove the unwanted blobs from the image completely. This function extracts all connected components (objects) from the given image, where the area of the objects is in the specified range and returns output image containing only the connected objects.

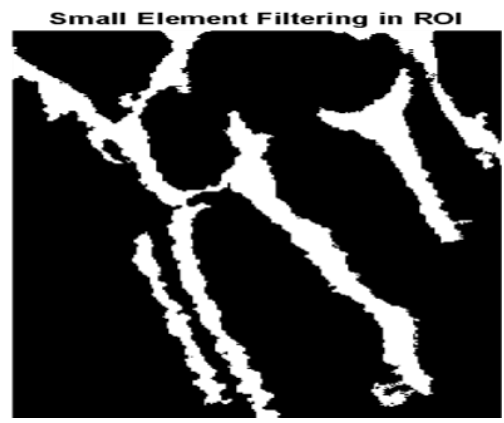

iv.

Fig.13 Small Element Filtering

The net obtained vein pattern is morphologically thinned to 1bit pattern using bwmorph function.It removes pixels on the boundaries of objects, preserves the orientation of the objects with respect to the image and retains their connected components. The remaining pixels form the 1-bit image skeleton.After skeletonization, we have deduced the vein pattern in the form of 1-bit lines that can be further processed to obtain the ending points and the branching points. These points in fact would account for the features that would be tested with the matching algorithm.

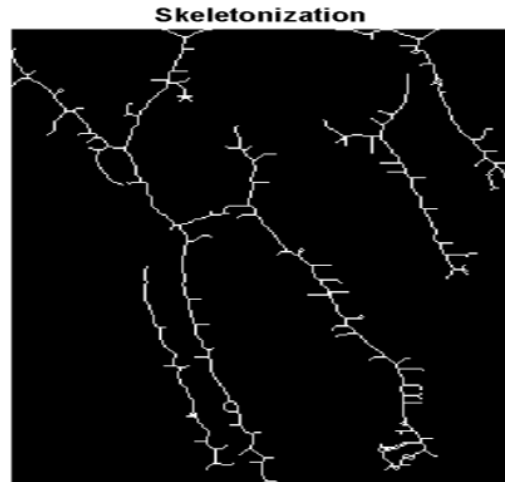

Fig.14 Skeletonization

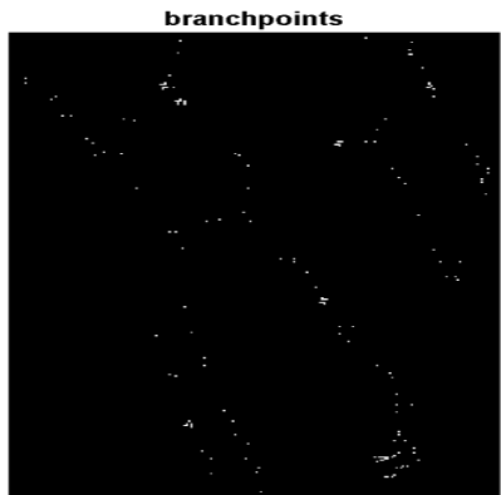

Fig. 15 Branch Points

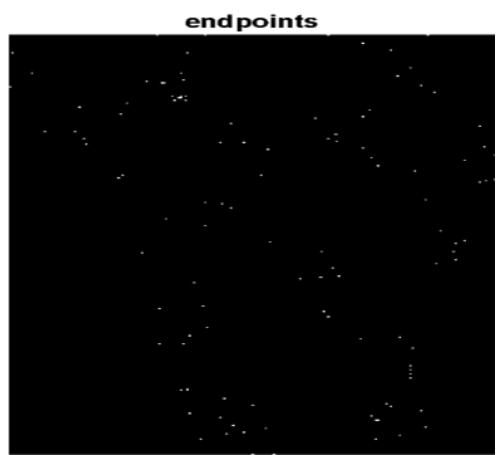

Fig. 16 End Points

\section{v. DELAUNAY'S TRIANGULATION}

The next step after obtaining Endpoints \& Branchpoints data is Triangulation. We have used Delaunay's Triangulation method which forms triplets with respect to the adjacent points in the neighbourhood area. Concept of using this feature for matching is taken from [1]. It reduces the number of narrow triangles and does not depend on vertex ordering. Delaunay's triangulation ensures that the circumcircle associated with each triangle contains no other point in its interior [22]. Triangulation helps in representation of points in a better medium on which the matching algorithm can be applied.

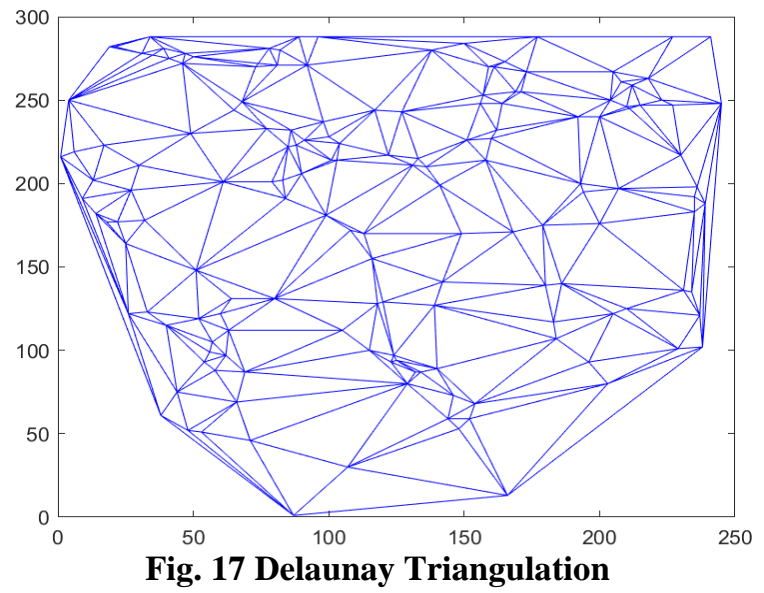

\section{F. MATCHING ALGORITHM}

Local \& Binary Features Detection, Extraction \& Matching is performed on the triangulated images together which constitute as Matching Algorithm using MATLAB functions [3][14]. For feature detection we have used Speeded-Up Robust Features (SURF) \& Binary Robust Invariant Scalable Key points (BRISK) algorithms. SURF algorithm is a local descriptor and works using local gradient computations. BRISK algorithm is a binary descriptor that relies on pairs of local intensity differences.For feature extraction we used Histogram of Gradients algorithm (HOG) and compared it with the extraction method based on collecting the descriptors from pixels surrounding an interest point.MatchFeatures function of MATLAB computes the Hamming distance between the matching features.

Published By:

Blue Eyes Intelligence Engineering \& Sciences Publication

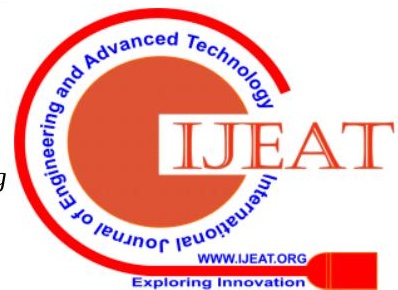


Human Identification Based on Hand Dorsal Vein Pattern using BRISK \& SURF Algorithm

The Matching percentage is calculated after computing a median distance of all the matched features. We have used Metric parameter along with Sum of absolute Differences (SAD).

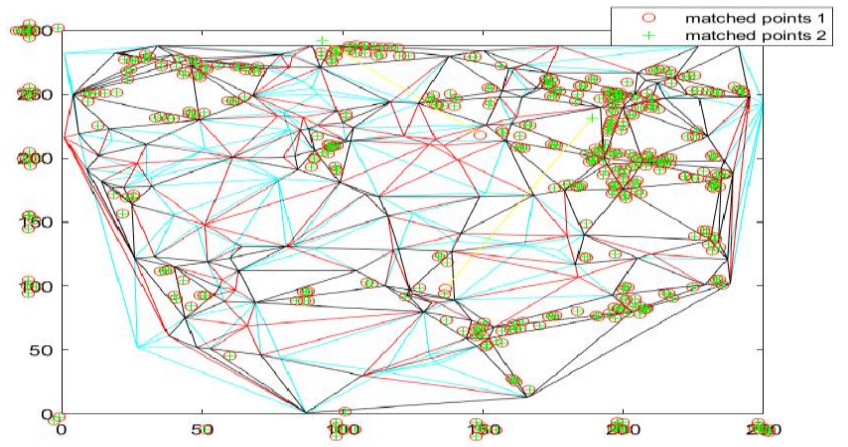

Fig.18 Matching with SURF and HOG

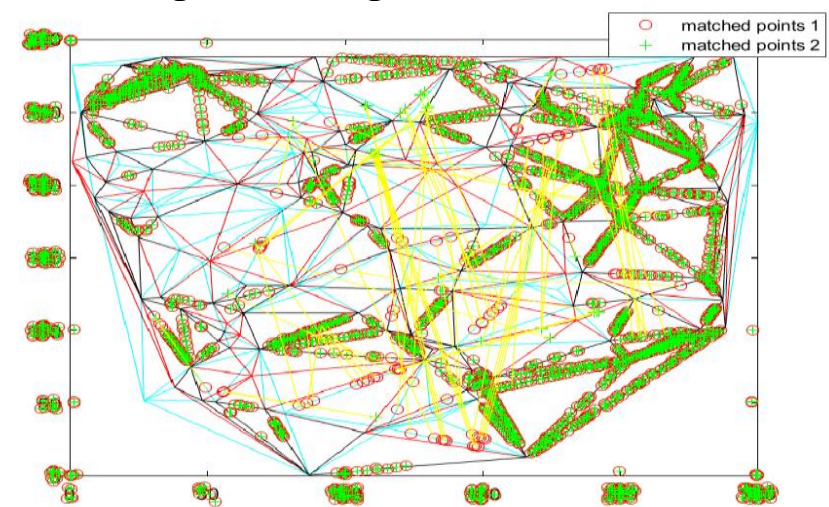

Fig. 19 Matching with BRISK and HOG

The above figures represent the pictorial representation of matching algorithms SURF \& BRISK respectively.

\section{RESULTS}

TABLE-I: Matching Percentage

\begin{tabular}{|c|c|c|}
\hline--- & BRISK & SURF \\
\hline Without HOG & $93.85 \%$ & $98.23 \%$ \\
\hline & & \\
With HOG & $99.72 \%$ & $99.51 \%$ \\
\hline
\end{tabular}

BRISK algorithm performs better than SURF algorithm along with extraction method based on HOG whereas using Local Gradient extraction method results in better accuracy of matching for SURF features.The database which we have used has a total 919 images of 155 persons (including male and female), in which one person has 6 images (3 NIR (near infrared) images of left hand and 3 NIR (near infrared) images of right hand).FAR is defined as the ratio of number of false acceptances to the total number of identification attempts. FRR is defined as the ratio of number of false rejections to the total number of identification attempts [3][14].The Table 2 represents the FAR and FRR of the first 50 persons calculated on threshold varying from 40 to 90 with an interval of 10.

TABLE-II: FAR and FRR of first 50 persons

\begin{tabular}{|c|c|c|c|}
\hline S. No. & $\begin{array}{c}\text { Threshold } \\
\text { (\%) }\end{array}$ & FAR & FRR \\
\hline 1 & 40 & 0.0684 & 0.0467 \\
\hline 2 & 50 & 0.0349 & 0.0498 \\
\hline
\end{tabular}

\begin{tabular}{|l|l|l|l|}
\hline 3 & 60 & 0.0142 & 0.0528 \\
\hline 4 & 70 & 0.0078 & 0.0967 \\
\hline 5 & 80 & 0.0026 & 0.1762 \\
\hline 6 & 90 & 0.0000 & 0.1867 \\
\hline
\end{tabular}

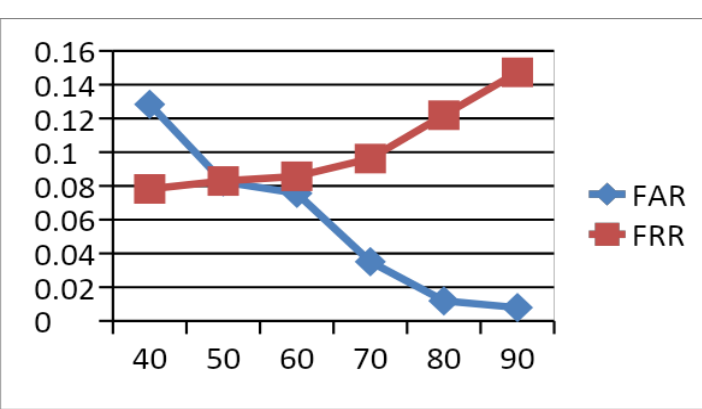

Fig.20 FAR and FRR vs Threshold \% graph of 50 persons

The Table 3 represents the FAR and FRR of the first 100 persons calculated on threshold varying from 40 to 90 with an interval of 10.

TABLE-III: FAR and FRR of first 100 persons

\begin{tabular}{|c|c|c|c|}
\hline S. No. & $\begin{array}{c}\text { Threshold } \\
\mathbf{( \% )}\end{array}$ & FAR & FRR \\
\hline 1 & 40 & 0.0976 & 0.0593 \\
\hline 2 & 50 & 0.0769 & 0.0658 \\
\hline 3 & 60 & 0.0438 & 0.0729 \\
\hline 4 & 70 & 0.0193 & 0.1137 \\
\hline 5 & 80 & 0.0093 & 0.1434 \\
\hline 6 & 90 & 0.0009 & 0.1621 \\
\hline
\end{tabular}

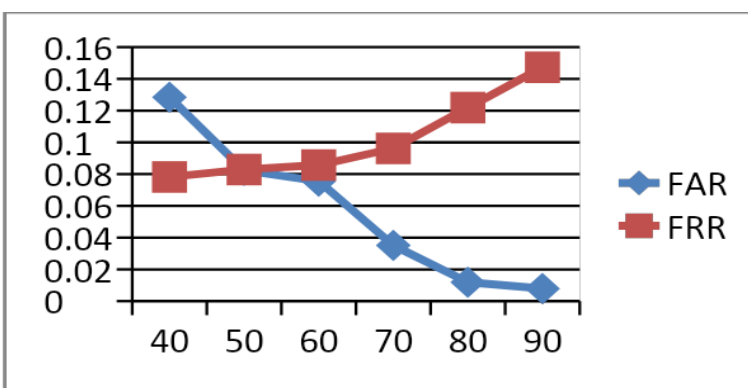

\section{Fig.21 FAR and FRR vs Threshold \% graph of 100 persons}

The Table 4 represents the FAR and FRR of all the 155 persons data present in the dataset [18] calculated on threshold varying from 40 to 90 with an interval of 10 .

TABLE-IV: FAR and FRR of first 155 persons

\begin{tabular}{|c|c|c|c|}
\hline S. No. & $\begin{array}{c}\text { Threshold } \\
(\mathbf{\%})\end{array}$ & FAR & FRR \\
\hline 1 & 40 & 0.1284 & 0.0782 \\
\hline 2 & 50 & 0.0824 & 0.0829 \\
\hline 3 & 60 & 0.0756 & 0.0857 \\
\hline 4 & 70 & 0.0351 & 0.0962 \\
\hline 5 & 80 & 0.0119 & 0.1219 \\
\hline 6 & 90 & 0.0079 & 0.1473 \\
\hline
\end{tabular}

Published By: Blue Eyes Intelligence Engineering \& Sciences Publication

2173 (C) Copyright: All rights reserved.

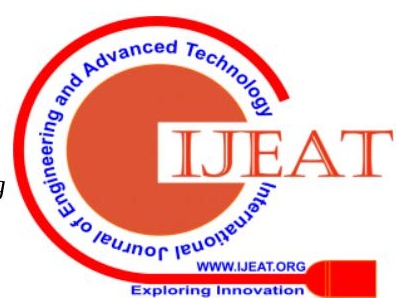




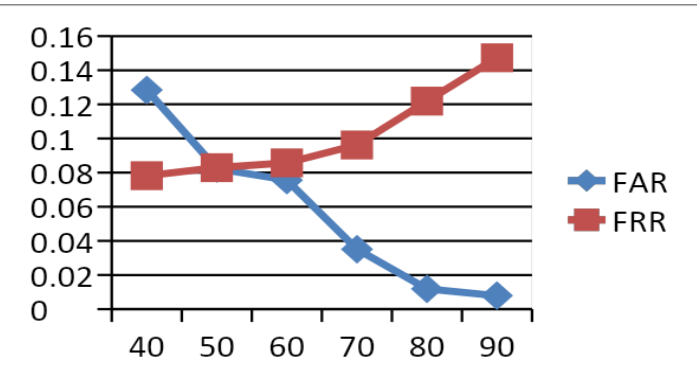

Fig.22 FAR and FRR vs Threshold \% graph of 155 persons

\section{CONCLUSION AND DISCUSSION}

From Table 1 we concluded that matching percentage is maximum for combination of BRISK and HOG with an outstanding result of $99.72 \%$ closely followed by SURF and HOG feature with $99.51 \%$. These results are better than SIFT algorithm and CNN algorithm [3][17]. Also, FAR and FRR graph come out to be desirable in nature. From Table 2, Table 3 and Table 4 we can conclude that as we increase the threshold percentage FAR decreases and FRR increases. We can also conclude that with increasing number of sample sizes both FAR and FRR increases. There is a lot of scope in future work in continuation with this research. First is to make a better feature extraction algorithm which can make matching percentage almost the same for each matching algorithm discussed in this paper. Second is comparing a healthy person vein pattern with an infected person from diseases such as malaria, cholera, diarrhoea etc. Third one is a multi-stage biometric system in which two or more than two biometric systems are used in succession to identify a person. This can be helpful in increasing the accuracy of the system as whole as we are using more than 2 biometric systems for authentication purposes.

\section{REFERENCES}

1. Sumit Kulkarni, Manali Pandit, "Biometric Recognition System based on Dorsal Hand Veins", International Journal of Innovative Research in Science, Engineering and Technology, ISSN(Online): 2319-8753, Vol. 5, Issue 9, pg. 18899-18905, September 2016

2. Humairah Hamid, V. K. Narang, Dr. Priti Singh, "Dorsal Hand Vein Analysis for Security Systems", International JournalofEngineeringandTechnology(IJET), e-ISSN:0975-4024, DOI:10.21817/ijet/2017/v9i4/170904044, Vol 9, No. 4, pg. 3075-3080, Aug-Sep 2017

3. Tarshi Jain, Rajendra Kumar, Ram Chandra Singh, "An Algorithm for Determining SIFT Matching Score for Dorsal Vein Recognition System", International Journal of Innovative Technology and Exploring Engineering (IJITEE), ISSN: 2278-3075, Volume-8, Issue-10S, pg. 468-471, August 2019

4. B. M. Sontakke, V. T. Humbe, P. L. Yannawar, "Automatic ROI Extraction and Vein Pattern Imaging of Dorsal Hand Vein Images", International Journal for Science and Advance Research in Technology (IJSART), ISSN [ONLINE]: 2395-1052, Volume 4 Issue 3, pg. 16781683, MARCH 2018

5. Tanya Shree, Ashwini Raykar, Pooja Jadhav, "Dorsal Palm Vein Engineering and Technology (IRJET), e-ISSN: 2395 -0056, Volume: 03 Issue: 06, pg. 2188-2192, June-2016

6. Marlina Yakno, Junita Mohamad-Saleh, Bakhtiar Affendi Rosdi, "New Technique for Larger ROI Extraction of Hand Vein Images", Pattern Based Recognition System", International Research Journal of

International Conference on Bio Signal Analysis, Processing and Systems (ICBAPS), 978-1-4799-6879-4/15/\$31.00, DOI:10.1109/ICBAPS.2015.7292223 pg. 82-87, May-2015

7. Dipti Verma, Dr. Sipi Dubey, "Processing and Enhancement of Palm Vein Image in Vein Pattern Recognition System", International Journal of Computer Science and Mobile Computing (IJCSMC), ISSN 2320088X, Vol. 4, Issue. 4, pg.137-141, April 2015

8. Ignacio Irving Morales-Montiel, J. Arturo Olvera-López, Manuel Martín-Ortíz, and Eber E. Orozco-Guillén, "Hand Vein Infrared Image Segmentation for Biometric Recognition", Research in Computing Science 80 (2014), pg. 55-66, rec. 2014-03-28; acc. 2014-10-30

9. M. Usman Akram, Hassan Moatasam Awan, Abdullah Amaan Khan, "Dorsal Hand Veins Based Person Identification", Image Processing Theory, Tools and Applications, 978-1-4799-6463-5/14/\$31.00 (C2014 IEEE

10. Ricardo Janes, Augusto Ferreira, Brand ao J'unior, “A Low-Cost System for Dorsal Hand Vein Patterns Recognition Using Curvelets", 2014 First International Conference on Systems Informatics, Modelling and Simulation, DOI 10.1109/SIMS.2014.17 pg. 37-42, 978-0-7695-51982/14 \$31.00 (C) 2014 IEEE

11. P. Ramsoful, M. Heenaye-Mamode Khan, "Feature extraction techniques for dorsal hand vein pattern", Third International Conference on Innovative Computing Technology (INTECH 2013), pp. 49-53.doi: 10.1109/INTECH.2013.6653722, London, 2013

12. Zahra Honarpisheh, Karim Faez, "An Efficient Dorsal Hand Vein Recognition Based on Firefly Algorithm", International Journal of Electrical and Computer Engineering (IJECE), ISSN:2088-8708, Vol 3, No. 1, pg. 30-41, February 2013

13. Nisha Charaya, Priti Singh, "Human Authentication Based on Dorsal Hand Veins: A Review", International Journal of Pure and Applied Mathematics, ISSN: 1314-3395, Volume 119, No. 16, pg. 2175-2185, 2018

14. Rajendra Kumar, Ram Chandra Singh, Ashok Kumar Sahoo, "SIFT based Dorsal Vein Recognition System for Cashless Treatment through Medical Insurance", International Journal of Innovative Technology and Exploring Engineering (IJITEE), ISSN: 2278-3075, Volume-8, Issue-10S, pg. 444-451, August 2019

15. G. Sathish S. V. Saravanan, S. Narmadha, S. U. Maheswari, "Personal Authentication System using Hand Vein Biometric.", International Journal of Computer Technology and Applications, vol. 3(1), pg. 383391, Jan-Feb 2012.

16. Abdul Kareem Z. Mohammed, Jamila H. Saud, "Feature Extraction from hand Dorsal Veinand Palmprint", International Journal of Advanced Research in Computer Engineering \& Technology (IJARCET), Volume 08, Issue 03, pg. 96-101, March 2019.

17. M. Rajalakshmi, V. Ganapathy, R. Rengaraj, "Palm-Dorsal Vein Pattern Authentication Using Convoluted Neural Network (CNN)", International Journal of Pure and Applied Mathematics (IJPAM), ISSN: 1314-3395 (on-line version), Volume 116 No. 23, pg. 525-532,2017

18. Dataset:https://www.researchgate.net/publication/338117068_SUAS_D orsal_Hand_Vein_Database

19. C. Premavathi, P. Thangaraj, "Efficient Hand-dorsa Vein Pattern Recognition using KNN classification with Completed histogram CB in TP Feature Descriptor", International Journal of Recent Technology and Engineering (IJRTE), ISSN: 2277-3878, Volume-7 Issue-4S, pg. 50-55, November 2018.

20. Ranjith Kumar M, Meenakshi, Krishnan, Deepika G, Rajesh Muthu, C. Rani, Karthikeyan B, "An Open Source Contact-Free Palm Vein Recognition System", International Journal of Advances in Applied Sciences (IJAS), ISSN: 2252-8814, Vol. 6, No.4, pg. 319-324, December 2017.

21. Shaimaa Ahmed Elsaid, Haifa Alharthi, ReemAlrubaia, Sarah Abutile, Rawan Aljres, AmalAlanazi, Alanoud Albrikan. "Chapter 12 ArabicReal-Time License Plate Recognition System",Advances in Data Science, Cyber Security and IT Applications. ICC 2019. Communications in Computer and Information Science, Springer Science and Business Media LLC, ISBN: 978-3-030-36368-0, Vol.1098, pg. 126-143, December 2019

22. MATLAB DOCUMENTATION HELP https://in.mathworks.com/help/

Published By:

Blue Eyes Intelligence Engineering \& Sciences Publication 
Human Identification Based on Hand Dorsal Vein Pattern using BRISK \& SURF Algorithm

\section{AUTHOR'S PROFILE}

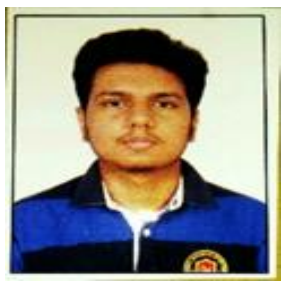

Aryan Singh, is pursuing B. Tech with majors in Electronics \& Communication Engineering from Delhi Technological University (Formerly Delhi College of Engineering), Delhi currently a Final year student. His current research interests include Biometric Systems, Signal Processing, Digital Image Processing and Embedded Systems

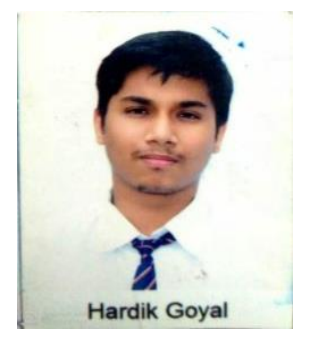

Hardik Goyal was born in Bulandshar, Uttar Pradesh, India in 1998. Currently he is studying Bachelors in Technology in Electronics and Communication Engineering from Delhi Technological University, (Formerly Delhi College of Engineering). $\mathrm{He}$ is a kind and peace-loving person. His research interests include Image Processing, Signal Processing, Biometric system, Data

Analytics and Digital Design Logic.

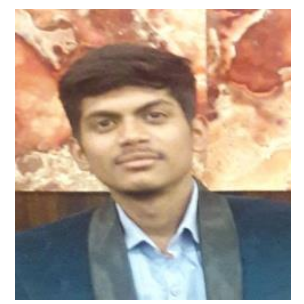

Amar is pursuing Bachelors in Technology in Electronics and Communication Engineering from Delhi Technological University(Formerly Delhi College of Engineering), Delhi. He is also an Abhay Anand Super 30 Alumnus. His current area of interests includes Biometric Systems, Digital Image Processing, Signal Processing, Digital Design and VLSI.

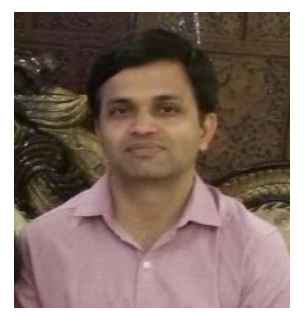

Ajai Kumar Gautam, is Assistant Professor of Electronics and Communication Engineering at Delhi Technological University (Formerly Delhi College of Engineering), Delhi. He had obtained Bachelors in Technology in Electronics Engineering from HBTI Kanpur, Masters in Technology in Electronics and Communication Engineering from Delhi University and Ph.D.(P) from Delhi Technological University. He has published two research papers in International Conferences. His research interests include Digital system \&Design, Microprocessors, Image processing and Embedded system. 